\title{
A neurometabolite study of chronic daily head- ache in patients with systemic lupus erythemato- sus using magnetic resonance spectroscopy: comparison with fibromyalgia patients and healthy controls
}

\author{
Chang-Nam Son ${ }^{1,}$, Sang-Hyon Kim ${ }^{1,}$, Hyuk Won Chang ${ }^{2}$, and Ji-Min Kim ${ }^{1}$
}

${ }^{1}$ Division of Rheumatology, Department of Internal Medicine, ${ }^{2}$ Department of Radiology, Keimyung University Dongsan Medical Center, Daegu, Korea

Received: June 29, 2015 Revised : August 25, 2015 Accepted: August 26, 2015

\section{Correspondence to}

Ji-Min Kim, M.D.

Division of Rheumatology,

Department of Internal Medicine,

Keimyung University Dongsan

Medical Center, 56 Dalseong-ro,

Jung-gu, Daegu 41931, Korea

Tel: $+82-53-250-7474$

Fax: +82-53-250-7434

E-mail: okjimin@dsmc.or.kr

*These authors contributed equally to this work.
Background/Aims: Neuropsychiatric systemic lupus erythematosus (SLE) includes a broad spectrum of neurologic and psychiatric manifestations. One of the most commonly observed neuropsychiatric symptoms is headache. However, the lack of specific clinical distinctions for headache in SLE has made it difficult to elucidate its pathophysiology. The aim of this study is to evaluate the neurometabolic changes using Proton Magnetic Resonance Spectroscopy ( $\left.{ }^{1} \mathrm{H}-\mathrm{MRS}\right)$ in patients with SLE who suffer from chronic daily headache (CDH).

Methods: SLE and fibromyalgia patients with $\mathrm{CDH}$ and healthy controls were recruited ( $\mathrm{n}=9, \mathrm{n}=5$, and $\mathrm{n}=6$, respectively). ${ }^{1} \mathrm{H}$-MRS metabolite ratios were evaluated in bilateral basal ganglia (BG) and bilateral peritrigonal white matter (PWM). Results: ${ }^{1} \mathrm{H}$-MRS showed a significantly decreased $\mathrm{N}$-acetylaspartate (NAA)/creatine $(\mathrm{Cr})$ ratio in right $\mathrm{BG}$ in SLE patients with $\mathrm{CDH}$ compared to fibromyalgia patients with $\mathrm{CDH}$ and normal controls ( $p=0.029$ and $p=0.020$, respectively). Left PWM NAA/Cr and choline/Cr ratios in SLE patients with CDH were lower than those in fibromyalgia patients with $\mathrm{CDH}(p=0.019$ and $p=0.029$, respectively).

Conclusions: This study suggests the possibility that CDH in patients with SLE might be associated with neuronal dysfunction and neurometabolic changes.

Keywords: Proton magnetic resonance spectroscopy; Lupus erythematosus, systemic; Headache disorders

\section{INTRODUCTION}

Systemic lupus erythematosus (SLE) is a chronic autoimmune disease mainly affecting the young age group including women of childbearing age. Approximately 2/3 of SLE patients have neuropsychiatric symptoms [1]. Neuropsychiatric systemic lupus erythematosus (NPSLE) includes a broad spectrum of neurologic and psychiatric manifestations. Headache is one of the most common symptoms of SLE patients but the relatedness to SLE is still controversial [2]. Chronic daily headache (CDH) is defined as a primary headache occurring at least 15 days per month and it is one of the most common problems in a headache clinic [3]. CDH patients do not respond 
well to medical treatment and take medical services repetitively; hence, curing $\mathrm{CDH}$ is very important from a therapeutic viewpoint.

Proton Magnetic Resonance Spectroscopy ( $\left.{ }^{1} \mathrm{H}-\mathrm{MRS}\right)$ is a non-invasive test that can demonstrate changes in neuronal metabolites [4]. In recent years, many studies using ${ }^{1} \mathrm{H}$-MRS have been actively conducted in NPSLE patients and the recommendation for NPSLE management proposed by the European League Against Rheumatism (EULAR) stated that if conventional magnetic resonance imaging (MRI) is normal and signs or symptoms cannot be explained, ${ }^{1} \mathrm{H}-\mathrm{MRS}$ can be considered $[4,5]$. However, most studies used ${ }^{1} \mathrm{H}-\mathrm{MRS}$ to compare patients with minor NPSLE versus patients with major NPSLE or they compared the SLE patients according to the existence of NPSLE [4]. However, no study has been conducted using MRS with a focus on specific symptoms, such as headache. Therefore, we aimed to evaluate neurometabolic changes by using ${ }^{1} \mathrm{H}-\mathrm{MRS}$ in SLE patients with $\mathrm{CDH}$, and to compare them with fibromyalgia patients with $\mathrm{CDH}$, or healthy controls.

\section{METHODS}

\section{Subjects}

SLE patients with CDH, fibromyalgia syndrome (FMS) patients with $\mathrm{CDH}$, and healthy controls who had no organic brain lesion were recruited into this study. The study participants with $\mathrm{CDH}$ were enrolled from among the patients who were registered at the Rheumatology Clinic of Keimyung University Dongsan Medical Center in Daegu. If the subject presented with headache for more than 15 days per month for longer than 3 months, he/she was defined as having CDH. A total of nine SLE patients without history of neuropsychiatric symptoms or current neuropsychiatric symptoms were Koreans and they fulfilled the 1997 American College of Rheumatology (ACR) revised criteria for the classification of SLE [6]. Furthermore, patients who did not have secondary FMS and any other comorbidity or history of medication that could cause a headache were selected. We reviewed data obtained from the subjects according to age, gender, concomitant treatment, disease duration, antinuclear antibody, extractable nuclear antigen antibody, and anti-phospholipid antibody. The patients underwent required blood tests for assessment of the disease activity by the Systemic Lupus Erythematosus Disease Activity Index (SLEDAI) [7].

To compare the MRS among SLE patients, FMS patients, and healthy controls, we recruited five age- and sex-matched FMS patients and six age- and sex-matched healthy subjects as the control group. A total of five FMS patients were also Korean nationals, and they met the 2010 ACR criteria for FMS [8]. We recruited SLE and FMS patients with $\mathrm{CDH}$ who were aged 18 years or older and who had received regular treatment for 3 months prior to study enrollment. Patients who had diabetes, stroke, renal insufficiency, and alcohol or substance abuse were excluded from the study. Healthy controls who did not have any disease that could cause a headache and who were not taking any drugs were recruited into this study. This study used a cross-sectional design.

\section{Proton Magnetic Resonance Spectroscopy}

${ }^{1} \mathrm{H}$-MRS was performed with a single voxel technique using point-resolved spectroscopy sequence with the following parameters: repetition time $=1,500 \mathrm{~ms}$, echo time $=30 \mathrm{~ms}$, number of excitations $=$ one, and $8 \mathrm{~cm}^{3}(2 \times$ $2 \times 2 \mathrm{~cm}$ ) voxels of interest (VOIs). Four VOIs were right and left basal ganglia (BG) and right and left peritrigonal white matter (PWM) because these VOIs are the most selected areas in the previous studies [4]. The total parameter scan time was 3 minutes 42 seconds for each VOI. The metabolite concentrations of $\mathrm{N}$-acetylaspartate (NAA), myoinositol (mI), choline (Cho), and creatine (Cr) were analyzed. The NAA/Cr, Cho/Cr, NAA/Cho, mI/Cr, and NAA/mI ratios were calculated (Fig. 1).

\section{Statistical analysis}

All statistical analyses were performed using SPSS version 20.0 (IBM Co., Armonk, NY, USA). Results are presented as mean \pm SD unless specified otherwise. The non-parametric Kruskal-Wallis test was used for between-group comparisons. The Bonferroni correction was applied to multiple comparisons within each variable. $p$ values less than 0.05 were considered statistically significant.

\section{Ethics statement}

This study was approved by the Institutional Review Board (IRB) of Keimyung University Dongsan Medical 

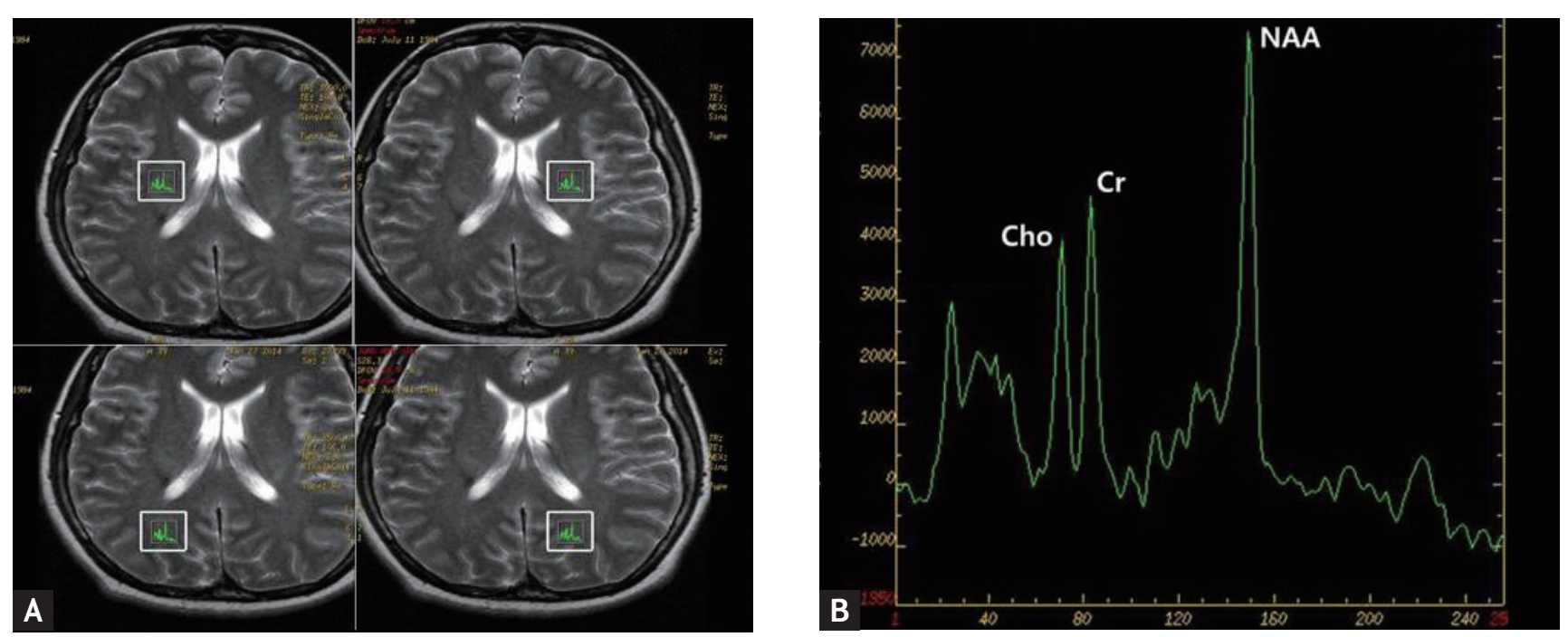

Figure 1. Images illustrating voxel placement at the level of the basal ganglia and the peritrigonal white matter (A) and the spectral pattern of lupus patient with chronic daily headache in basal ganglia (B). NAA, N-acetylaspartate; Cr, creatine; Cho, choline.

Center (IRB No. 2013-05-031). Written informed consent was obtained from all participants and confirmed by the board.

\section{RESULTS}

Table 1 presents the clinical and laboratory data of SLE patients enrolled in the study. Eight out of the nine SLE patients with CDH had SLEDAI $<6$, indicating low disease activity. Two SLE patients had antiphospholipid antibody syndrome. Three out of the nine SLE patients had lupus nephritis. The median dose of corticosteroids was $5.6 \mathrm{mg} /$ day. Eight patients were taking hydroxychloroquine, and two patients were taking mycophenolate mofetil. Table 2 shows the results of ${ }^{1} \mathrm{H}$-MRS measurements in SLE patients, FMS patients, and healthy controls. The mean age was not different among groups (41.7 $\pm 13.7,43.8 \pm 7.6$, and $31.2 \pm 6.2$ years for SLE patients with $\mathrm{CDH}$, FMS patients with $\mathrm{CDH}$, and healthy controls, respectively). The NAA/Cr ratio in right BG of patients with SLE, FMS, and healthy controls was $1.54 \pm 0.05,1.62$ \pm 0.07 , and $1.61 \pm 0.05$, respectively. Even after applying Bonferroni correction, the results showed a significantly decreased NAA/Cr ratio in right BG in SLE patients with $\mathrm{CDH}$ compared to fibromyalgia patients with $\mathrm{CDH}$ and normal controls $(p=0.029$ and $p=0.020$, respectively). However, there were no significant differences in the
NAA/Cr ratio in left BG among the three groups. Left PWM NAA/Cr and Cho/Cr ratios in SLE patients with $\mathrm{CDH}$ were significantly lower than those in FMS patients with $\mathrm{CDH}(p=0.019$ and $p=0.029$, respectively). Contrary to our thoughts, left PWM NAA/Cr and Cho/ Cr ratios in FMS patients with $\mathrm{CDH}$ were significantly higher than those in healthy controls $(p=0.047$ and $p=$ 0.023 , respectively). There were no significant differences in $\mathrm{mI} / \mathrm{Cr}$ and $\mathrm{NAA} / \mathrm{mI}$ ratios among the three groups (data not shown).

\section{DISCUSSION}

NPSLE consists of 19 syndromes including neurologic and psychiatric problems that affect the central nervous system (CNS) and the peripheral nervous system [1]. Among these problems, headache is the most common symptom which is observed in migraine, tension, benign intracranial hypertension, and non-specific intractable headache [9]. There is no standardized method to assess whether headache is related to lupus. Headache can occur due to secondary causes such as infection, medication, and hypertension [2]. In addition, it is controversial that headache in SLE patients can be considered as part of neurologic involvement and no correlation was found with disease activity [4].

Laboratory diagnosis of NPSLE is very difficult and 


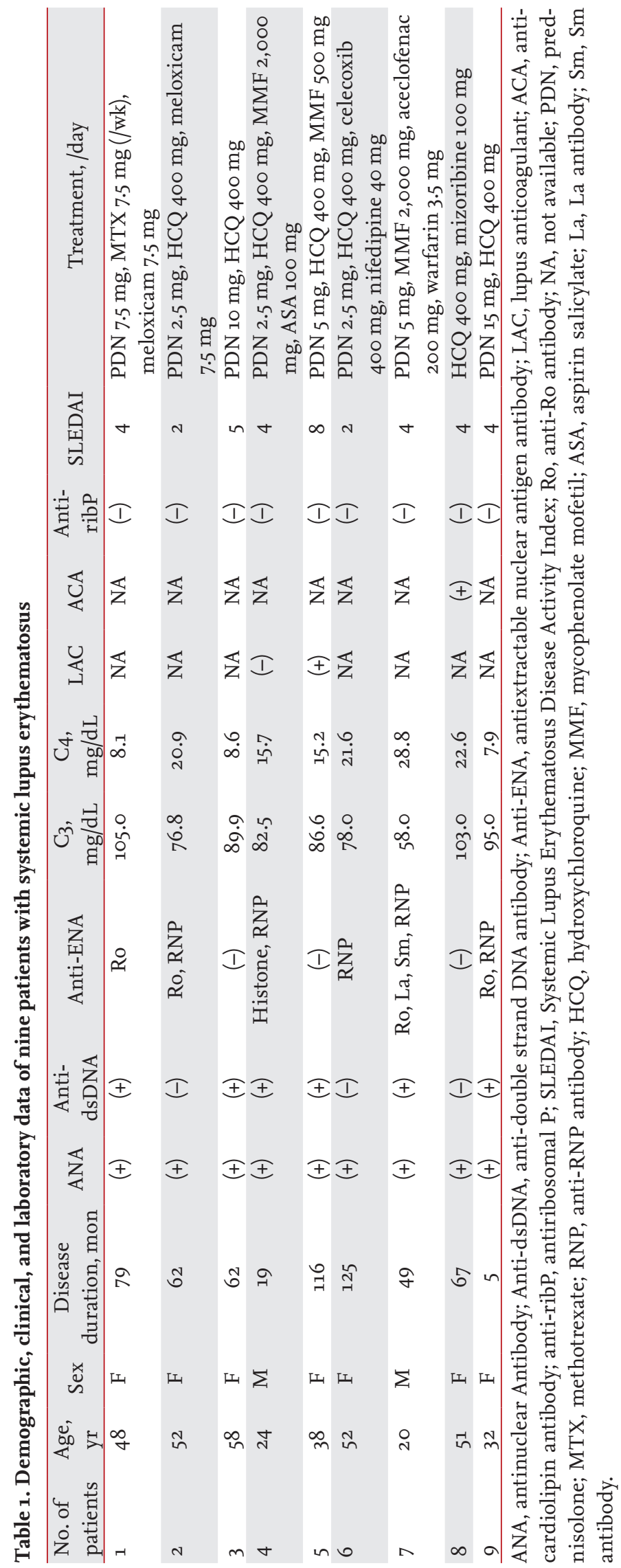

many cases have no correlation with disease activity of SLE [10]. Cerebrospinal fluid examination, electroencephalography, and MRI can be helpful in diagnosing NPSLE. If MRI shows normal or nonspecific changes, a more advanced imaging modality should be considered. According to the recommendation for management of NPSLE proposed by the EULAR, a more advanced imaging modality refers to quantitative MRI, single photon emission computed tomography, and positron emission tomography [5].

${ }^{1} \mathrm{H}-\mathrm{MRS}$ has been applied in physiological, biochemical, and pathological studies of the brain as a clinical tool to measure the amount of various metabolites in tissues non-invasively [4,11]. The proportion between metabolites reflects the molecular and cytological process. The NAA level is correlated with neuronal and axonal density, and $\mathrm{mI}$ is considered as a marker of gliosis. Cho refers to myelin content and it reflects membrane turnover, while $\mathrm{Cr}$ is used as a reference metabolite. As observed in studies on SLE using ${ }^{1} \mathrm{H}-\mathrm{MRS}$, the NAA level was decreased in both NPSLE patients and SLE patients when the Cho and $\mathrm{mI}$ levels were increased or decreased [4]. However, few studies analyzed the specific manifestations using ${ }^{1} \mathrm{H}$-MRS. Several studies showed that the Cho/Cr ratio was increased in patients with cognitive impairment [12,13].

In this study, ${ }^{1} \mathrm{H}-\mathrm{MRS}$ was conducted in patients who satisfied the definition of $\mathrm{CDH}$ [3]. In reality, it is difficult to recruit $\mathrm{CDH}$ controls without underlying diseases. FMS excluded generous organic causes of disease is often accompanied by headache. Because both FMS and $\mathrm{CDH}$ have pathophysiology for nonorganic chronic pain, we selected FMS patients with $\mathrm{CDH}$ as a control group. Our study result was consistent with other ${ }^{1} \mathrm{H}$-MRS studies conducted in other SLE patients. The results indicate that the NAA/Cr ratio in SLE patients was reduced more in the right BG compared to that in fibromyalgia patients and healthy controls. The metabolites in BG were measured in previous SLE studies $[14,15]$. Pragmatic trace metals are often deposited in the BG within the brain, and the BG are thought to be the most metabolically active part that is affected during hypoxic brain damage [15]. Furthermore, the NAA/Cr ratio was reduced more in the left PWM of SLE patients than fibromyalgia patients, but no significant difference was observed when compared to healthy controls. The 
Table 2. Comparison of the neurometabolite ratios in basal ganglia and peritrigonal white matter by ${ }^{1} \mathrm{H}-\mathrm{MRS}$

\begin{tabular}{|c|c|c|c|c|}
\hline Parameter & $\begin{array}{l}\text { Systemic lupus erythematosus } \\
\text { patients }\end{array}$ & Fibromyalgia patients & Healthy controls & $p$ value $^{a}$ \\
\hline No. of subjects & 9 & 5 & 6 & \\
\hline Age, yr & $41.7 \pm 13.7$ & $43.8 \pm 7.6$ & $31.2 \pm 6.2$ & 0.131 \\
\hline Sex, male/female & $2 / 7$ & $1 / 4$ & $1 / 5$ & \\
\hline \multicolumn{5}{|c|}{ Right basal ganglia } \\
\hline $\mathrm{NAA} / \mathrm{Cr}$ & $1.54 \pm 0.05$ & $1.62 \pm 0.07^{b}$ & $1.61 \pm 0.05^{b}$ & 0.041 \\
\hline Cho/Cr & $0.83 \pm 0.05$ & $0.87 \pm 0.04$ & $0.82 \pm 0.05$ & 0.157 \\
\hline NAA/Cho & $1.87 \pm 0.12$ & $1.86 \pm 0.12$ & $1.95 \pm 0.11$ & 0.327 \\
\hline \multicolumn{5}{|l|}{ Left basal ganglia } \\
\hline $\mathrm{NAA} / \mathrm{Cr}$ & $1.52 \pm 0.10$ & $1.57 \pm 0.09$ & $1.60 \pm 0.12$ & 0.399 \\
\hline Cho/Cr & $0.84 \pm 0.08$ & $0.86 \pm 0.05$ & $0.84 \pm 0.05$ & 0.794 \\
\hline NAA/Cho & $1.81 \pm 0.14$ & $1.82 \pm 0.11$ & $1.91 \pm 0.08$ & 0.177 \\
\hline \multicolumn{5}{|c|}{ Right peritrigonal white matter } \\
\hline NAA/Cr & $1.61 \pm 0.14$ & $1.71 \pm 0.11$ & $1.63 \pm 0.12$ & 0.521 \\
\hline Cho/Cr & $0.89 \pm 0.09$ & $0.97 \pm 0.09$ & $0.86 \pm 0.10$ & 0.202 \\
\hline NAA/Cho & $1.83 \pm 0.19$ & $1.77 \pm 0.15$ & $1.90 \pm 0.12$ & 0.381 \\
\hline \multicolumn{5}{|c|}{ Left peritrigonal white matter } \\
\hline $\mathrm{NAA} / \mathrm{Cr}$ & $1.61 \pm 0.11$ & $1.76 \pm 0.09^{b}$ & $1.60 \pm 0.10$ & 0.035 \\
\hline Cho/Cr & $0.88 \pm 0.11$ & $1.01 \pm 0.11^{b}$ & $0.82 \pm 0.11$ & 0.031 \\
\hline NAA/Cho & $1.84 \pm 0.11$ & $1.71 \pm 0.17$ & $1.97 \pm 0.27$ & 0.184 \\
\hline
\end{tabular}

Values are presented as mean $\pm \mathrm{SD}$.

${ }^{1}$ H-MRS, Proton Magnetic Resonance Spectroscopy; NAA, N-acetylaspartate; Cr, creatine; Cho, choline.

${ }^{a} p$ value between groups by Kruskal-Wallis test.

${ }^{\mathrm{b}}$ Statistically significant compared with systemic lupus erythematosus patients by applying Bonferroni correction after Kruskal-Wallis test $(p<0.05)$.

reason for the change in metabolites in the white matter area was that the commissural tract and a large area of the association tract were damaged thereby causing neuropsychiatric symptoms of NPSLE [16]. However, further studies are needed for elucidating the difference of left PWM NAA/Cr and Cho/Cr ratio between fibromyalgia patients and healthy controls because our study includes only a limited number of patients.

Our study had some limitations. First, the test was conducted only in two areas locally: BG and PWM. In other SLE studies, a number of areas such as periventricular, occipital, and frontal regions of white matter as well as the insular area were studied $[4,11,16,17]$. It has been reported that there was no difference in metabolites in the white matter regions between normal subjects and FMS patients and the NAA level in the hippocampus was reduced according to the ${ }^{1} \mathrm{H}-\mathrm{MRS}$ studies [18-20]. Second, the present study could not assess whether there were any changes in metabolites after treatment of CDH due to its cross-sectional design. If prospective studies are conducted, changes in metabolites due to disease activity or treatment can be used as a marker. Third, it was difficult to recruit a large number of SLE and FMS patients with $\mathrm{CDH}$ due to low prevalence of $\mathrm{CDH}$. Because of this limitation, we could not detect any differences according to the disease duration or treatment regimen. Therefore, a multicenter study should be performed so that a large number of patients can be recruited. However to date, no ${ }^{1} \mathrm{H}$-MRS studies have been conducted with respect to specific CNS manifestations such as $\mathrm{CDH}$. Fourth, SLE patients without CDH were not enrolled in this study. We did not compare the metabolites between SLE patients with CDH and SLE patients without CDH.

Based on the results of this study, we expect that additional studies including many cases with CNS manifestations will be performed in the future. Our study 
results provide useful evidence to shed light on the ${ }^{1} \mathrm{H}-\mathrm{MRS}$ study of specific CNS manifestations of NPSLE and pathophysiology of $\mathrm{CDH}$.

\section{KEY MESSAGE}

1. This study suggests the possibility that chronic daily headache (CDH) in patients with systemic lupus erythematosus might be associated with neuronal dysfunction and neurometabolic changes.

2. Our study results provide useful evidence to shed light on the Proton Magnetic Resonance Spectroscopy ( ${ }^{1} \mathrm{H}$-MRS) study of specific central nervous system manifestations of neuropsychiatric systemic lupus erythematosus and pathophysiology of $\mathrm{CDH}$.

\section{Conflict of interest}

No potential conflict of interest relevant to this article was reported.

\section{Acknowledgments}

This work was supported by the research promoting grant from the Keimyung University Dongsan Medical Center in 2013.

\section{REFERENCES}

1. The American College of Rheumatology nomenclature and case definitions for neuropsychiatric lupus syndromes. Arthritis Rheum 1999;42:599-608.

2. Cuadrado MJ, Sanna G. Headache and systemic lupus erythematosus. Lupus 2003;12:943-946.

3. Pascual J, Colas R, Castillo J. Epidemiology of chronic daily headache. Curr Pain Headache Rep 2001;5:529-536.

4. Appenzeller S, Costallat LT, Li LM, Cendes F. Magnetic resonance spectroscopy in the evaluation of central nervous system manifestations of systemic lupus erythematosus. Arthritis Rheum 2006;55:807-811.

5. Bertsias GK, Ioannidis JP, Aringer M, et al. EULAR recommendations for the management of systemic lupus erythematosus with neuropsychiatric manifestations: re- port of a task force of the EULAR standing committee for clinical affairs. Ann Rheum Dis 2010;69:2074-2082.

6. Hochberg MC. Updating the American College of Rheumatology revised criteria for the classification of systemic lupus erythematosus. Arthritis Rheum 1997;40:1725.

7. Bombardier C, Gladman DD, Urowitz MB, Caron D, Chang $\mathrm{CH}$. Derivation of the SLEDAI: a disease activity index for lupus patients. The Committee on Prognosis Studies in SLE. Arthritis Rheum 1992;35:630-640.

8. Wolfe F, Clauw DJ, Fitzcharles MA, et al. The American College of Rheumatology preliminary diagnostic criteria for fibromyalgia and measurement of symptom severity. Arthritis Care Res (Hoboken) 2010;62:600-610.

9. Unterman A, Nolte JE, Boaz M, Abady M, Shoenfeld Y, Zandman-Goddard G. Neuropsychiatric syndromes in systemic lupus erythematosus: a meta-analysis. Semin Arthritis Rheum 2011;41:1-11.

10. Bertsias GK, Boumpas DT. Pathogenesis, diagnosis and management of neuropsychiatric SLE manifestations. Nat Rev Rheumatol 2010;6:358-367.

11. Castellino G, Govoni M, Padovan M, Colamussi P, Borrelli M, Trotta F. Proton magnetic resonance spectroscopy may predict future brain lesions in SLE patients: a functional multi-imaging approach and follow up. Ann Rheum Dis 2005;64:1022-1027.

12. Brooks WM, Jung RE, Ford CC, Greinel EJ, Sibbitt WL Jr. Relationship between neurometabolite derangement and neurocognitive dysfunction in systemic lupus erythematosus. J Rheumatol 1999;26:81-85.

13. Kozora E, Arciniegas DB, Filley CM, et al. Cognition, MRS neurometabolites, and MRI volumetrics in non-neuropsychiatric systemic lupus erythematosus: preliminary data. Cogn Behav Neurol 2005;18:159-162.

14. Bae SK, Song JS, Park W. 1H-magnetic resonance spectroscopy for the early diagnosis of neuropsychiatric systemic lupus erythematosus. J Korean Rheum Assoc 2000;7:370380.

15. Lim MK, Suh CH, Kim HJ, et al. Systemic lupus erythematosus: brain MR imaging and single-voxel hydrogen 1 MR spectroscopy. Radiology 2000;217:43-49.

16. Zimny A, Szmyrka-Kaczmarek M, Szewczyk P, et al. In vivo evaluation of brain damage in the course of systemic lupus erythematosus using magnetic resonance spectroscopy, perfusion-weighted and diffusion-tensor imaging. Lupus 2014;23:10-19.

17. Cagnoli P, Harris RE, Frechtling D, et al. Reduced insular 
glutamine and $\mathrm{N}$-acetylaspartate in systemic lupus erythematosus: a single-voxel (1)H-MR spectroscopy study. Acad Radiol 2013;20:1286-1296.

18. Fayed N, Garcia-Campayo J, Magallon R, et al. Localized $1 \mathrm{H}$-NMR spectroscopy in patients with fibromyalgia: a controlled study of changes in cerebral glutamate/glutamine, inositol, choline, and N-acetylaspartate. Arthritis Res Ther 2010;12:R134.
19. Petrou M, Harris RE, Foerster BR, et al. Proton MR spectroscopy in the evaluation of cerebral metabolism in patients with fibromyalgia: comparison with healthy controls and correlation with symptom severity. AJNR Am J Neuroradiol 2008;29:913-918.

20. Aoki Y, Inokuchi R, Suwa H. Reduced N-acetylaspartate in the hippocampus in patients with fibromyalgia: a meta-analysis. Psychiatry Res 2013;213:242-248. 